\title{
Expression of PANDA, LincRNA-p21, PUMA in lung tissues of lung cancer patients in the Xuanwei and non-Xuanwei areas of Yunnan Province
}

\author{
Kai-Yun Yang ${ }^{1,3}$, Zhi-Qiang Shen 1 , Yue-Feng He${ }^{2}$, Kushal Rizal ${ }^{3}$, Hui Tan ${ }^{3}$, An-Ning Chen ${ }^{3}$, Yun-Chao Huang ${ }^{3}$, \\ Guang-Qiang Zhao ${ }^{3}$, Yu-Jie Lei ${ }^{3}$ \\ ${ }^{1}$ Pharmacy College of Kunming Medical University, Chenggong, Kunming 650500, Yunnan, China. \\ ${ }^{2}$ Public Health College of Kunming Medical University, Chenggong, Kunming 650500, Yunnan, China. \\ ${ }^{3}$ The Third Affiliated Hospital of Kunming Medical University (Tumor Hospital of Yunnan), Kunming 650118, Yunnan, China.
}

Correspondence to: Prof. Zhi-Qiang Shen, Pharmacy College of Kunming Medical University, Yuhua District Spring Melt Road No. 1168, Chenggong, Kunming 650500, Yunnan, China. E-mail: 231833488@qq.com

How to cite this article: Yang KY, Shen ZQ, He YF, Rizal K, Tan H, Chen AN, Huang YC, Zhao GQ, Lei YJ. Expression of PANDA, LincRNA-p21, $P U M A$ in lung tissues of lung cancer patients in the Xuanwei and non-Xuanwei areas of Yunnan Province. J Cancer Metastasis Treat 2017;3:65-70.

Article history:

Received: 17-11-2016

Accepted: 22-02-2017

Published: 27-03-2017

Key words:

Lung cancer,

PANDA,

LincRNA-p21,

PUMA,

Xuanwei area

\begin{abstract}
Aim: To study the expression of PANDA, LincRNA-p21, and PUMA in lung tissue of patients with lung cancer from Xuanwei of Yunnan Province. Methods: Forty-five cases of lung cancer patients from Xuanwei and 42 lung cancer cases from non-Xuanwei were enrolled. Extraction of RNA was done using the Trizol kit. Real-time fluorescence quantitative PCR assay was done to obtain the relative expression. Results: Expressions of PANDA, LincRNA-p21, and PUMA in male and female patients or in squamous cell carcinoma and adenocarcinoma were not significantly different $(P>0.05)$. However, expression of LincRNA-p21 in Xuanwei patients was higher than non-Xuanwei patients $(P<0.05)$. Expression of PUMA in tumor tissue was lower than that in normal lung tissue $(P<0.05)$, and in Xuanwei patients was lower than non-Xuanwei patients $(P<0.05)$. In patients from non-Xuanwei regions, expression of LincRNA-p21 in patients with smoking index $>400$ was higher than in those $<400$ and non-smokers. Conclusion: Expressions of PANDA, LincRNA-p21, and PUMA in lung tissues have no gender differences or tissue specificity. High expression of LincRNA-p21 in Xuanwei patients may have relationship with cell damage caused by coal burning pollution in Xuanwei.
\end{abstract}

\section{INTRODUCTION}

Xuanwei is located in the northeast of Yunnan, China. In the east it is bordered by Panxian County of Guizhou, in the south by Zhanyi and Fuyuan Counties. Xuanwei has a population of $1,518,500$. It has an estimated 801,100 males and 717,400 females. The primary source of income in Xuanwei is from agriculture, coal

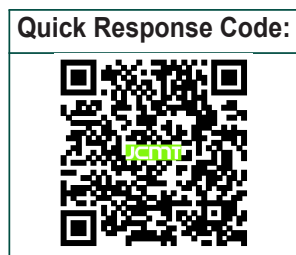


mining, and coal-related industries.

Xuanwei is an area with high deposits of coal and also has high incidence of lung cancer as well as high mortality rate from lung cancer. From 2004 to 2005, the mortality rate of lung cancer in Xuanwei was $91.30 / 10^{5}$, 2.26 times higher than that of Yunnan Province and 2.96 times higher than that of all of China. ${ }^{[1]}$ Current research suggests that the high incidence of lung cancer in Xuanwei is related to air pollution caused by bituminouscoal, with environmental factors perhaps accounting for $70 \%$ and $30 \%$ depending on susceptibility factors. ${ }^{[2]}$

Development of lung cancer is a complex biological, multistep process, which not only activates oncogenes and inactivates the tumor suppressor genes, but also has a close relation with imbalance of apoptosis. ${ }^{[3]}$ PANDA (DNA damage activates p21 related noncoding RNA), LincRNA-p21 (Cyclin-dependent kinase inhibitor 1a, CDKN1A/p21), and PUMA (p53 upregulated modulator of apoptosis) play an important role in p53-dependent mitochondrial apoptotic pathway when DNA is damaged. When DNA damage occurs, it activates expression of p53 and LincRNA-p21. In one aspect, the combination of p53 and LincRNA-p21 activates expression of PUMA, which then interacts with antiapoptotic members of the family tore lease Bax and Bak. Baxis embedded in the outer membrane of mitochondria, leading to increased mitochondrial outer membrane permeability. The release of cytochrome $\mathrm{C}$ apoptotic factors activates the caspase cascade, eventually leading to cell death. ${ }^{[4]}$ On the other hand, the combination of p53 and LincRNA-p21 activates expression of PANDA which in combination with Nuclear transcription factor $Y$ subunit alpha (NFYA) prevent apoptosis-related gene expression, thus inhibiting apoptosis. ${ }^{[5]}$

Our initial research found that smoke and ash of bituminous coal in Xuanwei contained large numbers of polyaromatic hydrocarbons and nano-sized quartz particles, which were also found in lung tissues of people with lung cancer in Xuanwei, which can lead to DNA damage as outlined earlier. We also found mutations and polymorphisms of mitochondrial DNA and miRNA in tissues of lung cancer patients from Xuanwei. By employing real-time quantitative fluorescence PCR techniques, expressions of PANDA, LincRNA-p21, and PUMA were examined in lung cancer tissues of patients from Xuanwei and non-Xuanwei areas. We also sought to investigate the relationship between PANDA, LincRNA-p21, PUMA and lung cancer risk in the Xuanwei area.

\section{METHODS}

\section{Objectives}

Forty-five patients from Xuanwei and 42 from nonXuanwei regions were recruited for surgery in the Department of Thoracic Surgery, the Third Affiliated Hospital of Kunming Medical University (Cancer Hospital of Yunnan) from March 2014 to December 2014. Details of the patients were obtained, including age, sex, personal habits, living condition, use of coal for household purposes, dietary habits, occupation, and economic conditions of the family. Chest X-ray, ultrasonography, CT scan, fiber optic bronchoscopy, and sputum cytology were done before surgery. All patients were chemotherapy-and radiotherapy-naive [Table 1].

Signed informed consents were obtained from all patients. Lung cancer tissues and normal lung tissues (5 $\mathrm{cm}$ away from tumor margins) was resected and preserved in RNA, later (Ambion, United States) in in vitro solution at a temperature of $4{ }^{\circ} \mathrm{C}$ for $8 \mathrm{~h}$ and then at $-80^{\circ} \mathrm{C}$ for medium or long term preservation. Clinical and pathological data were collected post operatively and the patients had regular follow-ups.

\section{Extraction of RNA}

Total extraction of RNA was done using the Triazol reagent kit (Invitrogen Company, USA) and reverse transcription was performed using the QuantScript RT kit (Tiangen Company, China). Using ABI7900HT (ABI Company, USA) according to established methods, three RNA (PANDA, LincRNA-p21, PUMA) and 2- $\triangle \mathrm{CT}$ value indicated the quantity of relative expression.

\section{Statistical analysis}

Statistical analysis was done using SPSS 17.0 software. After log transformation, normal distribution was analyzed. Comparison between the two groups was done using $t$-test and Spearman analysis of correlation was performed between the groups.

\section{RESULTS}

\section{The basic situation of cases}

A total of 45 patients with lung cancer were selected

Table 1: Inclusion and exclusion criteria

\begin{tabular}{ll}
\hline Inclusion criteria & Exclusion criteria \\
\hline Community: Han & $\begin{array}{l}\text { Silicosis, severe chronic obstructive } \\
\text { pulmonary disease, tuberculosis }\end{array}$ \\
$\begin{array}{l}\text { Primary carcinoma of } \\
\text { the lungs, non-small } \\
\text { cell lung cancer }\end{array}$ & $\begin{array}{l}\text { Surgical procedure: lobectomy, } \\
\text { segmentectomy, pneumonectomy }\end{array}$ \\
$\begin{array}{l}\text { Absence of concurrent } \\
\text { tumors }\end{array}$ & $\begin{array}{l}\text { No history of chemotherapy, } \\
\text { radiotherapy, targeted drug therapy }\end{array}$ \\
\hline
\end{tabular}


from Xuanwei and 42 patients with lung cancer were from non-Xuanwei regions. All patients were ethnic Han Chinese. Non-Xuanwei regions included Kunming, Yuxi, Baoshan, Dali, Chuxiong, Honghe, and other areas. Eighteen cases of squamous cell carcinoma and 27 cases of adenocarcinoma were from Xuanwei. Seventeen cases of squamous cell carcinoma and 25 cases of adenocarcinoma were from non-Xuanwei. There were 24 males and 21 females from Xuanwei and 24 males and 18 females from non-Xuanwei regions. Meanage of patients from Xuanwei was 54.91 \pm 9.62 years while in the non-Xuanwei group, the mean age was $58.33 \pm 10.13$ years. There were 21 patients from Xuanwei and 22 from non-Xuanwei who were smokers. Eighteen patients from Xuanwei consumed alcohol whereas 16 from non-Xuanwei did [Table 2].

Expressions of LincRNA-p21, PANDA, and $P U M A$ in Xuanwei and non-Xuanwei patients Expression of LincRNA-p21 in lung tissues of Xuanwei patients was increased $(P<0.05)$. There was no significant difference in expression of it between 2 groups of patients in lung cancer tissues and normal

Table 2: Comparison between the two groups

\begin{tabular}{lll}
\hline & Xuanwei & Non-Xuanwei \\
\hline$n$ & 45 & 42 \\
Squamous cell carcinoma & 18 & 17 \\
Adenocarcinoma & 27 & 25 \\
Male & 24 & 24 \\
Non-smoking & 3 & 2 \\
Smoking $<400$ & 7 & 9 \\
Smoking $>400$ & 14 & 13 \\
Female & 21 & 18 \\
Non-smoking & 21 & 18 \\
Smoking $<400$ & 0 & 0 \\
Smoking $>400$ & 0 & 0 \\
Age & $54.91 \pm 9.62$ & $58.33 \pm 10.13$ \\
Alcohol & 18 & 16 \\
\hline
\end{tabular}

*Smoking index: the number of cigarettes smoked per day $x$ number of years of smoking, high-risk groups $>400$ lung tissues $(P>0.05)$. There was no significant difference in the expression of PANDA in lung cancer tissue and normal lung tissue between the 2 groups $(P>0.05)$. Expression of PUMA in lung cancer tissue was lower than normal tissue $(P<0.05)$. Expression of PUMA in patients from Xuanwei was lower than that of non-Xuanwei patients, not only in cancer tissue, but also in normal lung $(P<0.05)$ [Tables 3 and 4 ].

Expression of LincRNA-p21, PANDA, and $P U M A$ in squamous cell carcinoma and adenocarcinoma

There was no significant difference in the expression of three genes in squamous cell carcinoma and adenocarcinoma $(P>0.05)$ [Table 5].

\section{Expression of LincRNA-p21, PANDA, PUMA in smokers and non-smokers}

The expression of LincRNA-p21, PANDA, PUMA in smokers and non-smokers was showed in Table 6.

Analysis of correlation in expression of LincRNA-p21, PANDA and PUMA in tissues of lung cancer patients from Xuanwei and nonXuanwei areas

LincRNA-p21 and PANDA in lung cancer tissues and normal lung tissues were characterized by positive correlation. PANDA with PUMA in lung cancer and normal lung tissue showed no corresponding correlation [Table 7].

Expression of LincRNA-p21, PANDA and PUMA in lung cancer patients from non-Xuanwei areas: LincRNA-p21 was positively correlated with PUMA in normal tissue, but not in lung cancer tissue. LincRNA-p21 and PANDA in lung cancer and normal lung tissue all showed positive correlation. PANDA was positively correlated with PUMA in normal lung tissue but not in lung cancers [Table 8].

Table 3: Expression of LincRNA-p21, PANDA, PUMA in males and females

\begin{tabular}{|c|c|c|c|c|}
\hline Gene & & Male & Female & $P$ \\
\hline \multirow{2}{*}{ LincRNA-p21 } & Xuanwei & $15.18 \pm 1.44$ & $15.19 \pm 1.65$ & 0.63 \\
\hline & Non-Xuanwei & $13.33 \pm 1.85$ & $13.01 \pm 1.05$ & 0.51 \\
\hline \multirow[t]{2}{*}{ PANDA } & Xuanwei & $14.36 \pm 1.65$ & $14.38 \pm 2.79$ & 0.88 \\
\hline & Non-Xuanwei & $14.14 \pm 1.23$ & $14.03 \pm 1.67$ & 0.85 \\
\hline \multirow[t]{2}{*}{ PUMA } & Xuanwei & $5.32 \pm 1.85$ & $5.23 \pm 1.43$ & 0.74 \\
\hline & Non-Xuanwei & $7.45 \pm 2.18$ & $7.34 \pm 2.42$ & 0.77 \\
\hline
\end{tabular}

Expression of the 3 genes in lung tissues of both males and females showed no statistical difference $(P>0.05)$

Table 4: Expression of LincRNA-p21, PANDA, PUMA in Xuanwei and non-Xuanwei patients

\begin{tabular}{|c|c|c|c|c|}
\hline Gene & & Xuanwei & Non-Xuanwei & $\boldsymbol{P}$ \\
\hline \multirow[t]{2}{*}{ LincRNA-p21 } & Cancer tissue & $15.12 \pm 1.27$ & $12.21 \pm 1.51$ & 0.03 \\
\hline & Normal tissue & $15.07 \pm 1.42$ & $12.19 \pm 1.62$ & 0.04 \\
\hline \multirow[t]{2}{*}{ PANDA } & Cancer tissue & $14.36 \pm 1.46$ & $14.08 \pm 1.27$ & 0.52 \\
\hline & Normal tissue & $14.44 \pm 1.55$ & $14.11 \pm 2.32$ & 0.47 \\
\hline \multirow[t]{2}{*}{ PUMA } & Cancer tissue & $5.14 \pm 1.37$ & $7.38 \pm 2.74$ & 0.01 \\
\hline & Normal tissue & $6.19 \pm 1.43^{*}$ & $8.82 \pm 2.89^{* *}$ & 0.01 \\
\hline
\end{tabular}

${ }^{\star} P=0.04$ (cancer tissue vs. normal tissue); ${ }^{\star \star} P=0.03$ (cancer tissue vs. normal tissue) 
Table 5: Expression of LincRNA-p21, PANDA and PUMA in squamous cell carcinoma and adenocarcinoma

\begin{tabular}{lllll}
\hline Gene & & Adenocarcinoma & Squamous cell carcinoma & $\boldsymbol{P}$ \\
\hline LincRNA-p21 & Xuanwei & $15.23 \pm 1.31$ & $15.09 \pm 1.53$ & 0.56 \\
PANDA & Non-Xuanwei & $12.31 \pm 1.73$ & $12.18 \pm 2.05$ & 0.58 \\
& Xuanwei & $14.38 \pm 1.64$ & $14.22 \pm 1.88$ & 0.74 \\
PUMA & Non-Xuanwei & $14.11 \pm 1.31$ & $14.01 \pm 2.15$ & 0.82 \\
& Xuanwei & $5.09 \pm 1.15$ & $5.33 \pm 1.84$ & 0.64 \\
\hline
\end{tabular}

Table 6: Expression of LincRNA-p21, PANDA, PUMA in smokers and non-smokers

\begin{tabular}{lllll}
\hline Gene & & Non-Smoker & Smoking $<\mathbf{4 0 0}$ & Smoking $>$ 400 \\
\hline LincRNA-p21 & Xuanwei & $15.19 \pm 1.01$ & $15.14 \pm 1.21$ & $15.16 \pm 2.02$ \\
PANDA & Non Xuanwei & $12.95 \pm 2.13$ & $13.16 \pm 1.73$ & $14.74 \pm 2.11^{*}$ \\
& Xuanwei & $14.37 \pm 1.21$ & $14.37 \pm 1.53$ & $14.38 \pm 1.74$ \\
PUMA & Non Xuanwei & $14.08 \pm 0.88$ & $14.15 \pm 1.29$ & $14.14 \pm 1.29$ \\
& Xuanwei & $5.31 \pm 1.47$ & $5.33 \pm 1.91$ & $5.32 \pm 1.12$ \\
\end{tabular}

${ }^{*} P<0.05$ (comparing non-smokers with smokers with smoking index $<400$ and $>400$ )

\section{DISCUSSION}

The Xuanwei region of Yunnan, China, has a high incidence of lung cancer. ${ }^{[2]}$ The characteristics of lung cancer in Xuanwei are: (1) the mortality rate of lung cancer in Xuanwei was 28.20/100,000 in 1973-1975, in 1990-1992 it was 40.29/100,000, and in 2004-2005 it was $83.28 / 100,000$, which were higher than the average of the entire Yunnan and the national average; (2) lung cancer caused death 10-15 years earlier than the national average age; (3) lung cancer incidence is high among female population whereas the sex ratio (male:female) is low in other places like Netherland (16.32), Sweden (4.15), China (2.01), whereas in it is 1.09. In the small town of Laibin in Xuanwei the sex ratio for the incidence of lung cancer is 0.87 , with females being affected more than males; (4) the incidence of lung cancer in Xuanwei area may be familial.

Indoor burning of bituminouscoal, leading to indoor air pollution, is considered to be the main reason for the high incidence of lung cancer in Xuanwei. Polyaromatic hydrocarbons (PAHs), nano silica particles and other substances in bituminouscoal dust and smoke are thought to be the causes of lung cancer. ${ }^{[6,7]}$ In 1970, the Chinese government implemented a policy at all levels to improve coal burning stoves which could lead to improvement of indoor air quality. Despite these efforts, lung cancer incidence is still high and with an increasing trend.

The development of lung cancer is a complex biological process which is multistep and multifactorial. It not only includes the activation and inactivation of oncogenes and tumor suppressor gene respectively, but also has close relationship with apoptosis. There are two pathways of apoptosis of which one is the activation of the apoptotic caspase through extracellular signaling while the other is release of cytochrome $\mathrm{C}$ and activation of apoptotic enzyme
Table 7: Expression of LincRNA-p21, PANDA and PUMA in lung cancer patients from Xuanwei

\begin{tabular}{llcc}
\hline \multirow{2}{*}{ Gene } & \multirow{2}{*}{ Tissue type } & \multicolumn{2}{c}{ Spearman correlation } \\
\cline { 3 - 4 } & & $\boldsymbol{R}$ & $\boldsymbol{P}$ \\
\hline LincRNA-p21 & Cancer tissue & 0.08 & 0.62 \\
and PUMA & Normal tissue & 0.12 & 0.33 \\
PANDA and & Cancer tissue & 0.07 & 0.58 \\
PUMA & Normal tissue & 0.14 & 0.28 \\
LincRNA-p21 & Cancer tissue & 0.81 & 0.00 \\
and PANDA & Normal tissue & 0.77 & 0.00 \\
\hline
\end{tabular}

Table 8: Expression of LincRNA-p21, PANDA and PUMA in lung cancer patients from non-Xuanwei areas

\begin{tabular}{llcc}
\hline \multirow{2}{*}{ Gene } & \multirow{2}{*}{ Tissue type } & \multicolumn{2}{c}{ Spearman correlation } \\
\cline { 3 - 4 } & & $\boldsymbol{R}$ & $\boldsymbol{P}$ \\
\hline LincRNA-p21 & Cancer tissue & 0.06 & 0.67 \\
and PUMA & Normal tissue & 0.35 & 0.04 \\
PANDA and & Cancer tissue & 0.08 & 0.62 \\
PUMA & Normal tissue & 0.42 & 0.00 \\
LincRNA-p21 & Cancer tissue & 0.84 & 0.00 \\
and PANDA & Normal tissue & 0.71 & 0.00 \\
\hline
\end{tabular}

through mitochondrial caspase. ${ }^{[8,9]}$ It is well known that repeated DNA damage can be carcinogenic, and tumor suppressor p53 increases as a response to DNA damage. Burning of coal produces numerous harmful pollutants and carcinogens which causes damage to DNA. At the molecular level, DNA damage is sensed by human protein kinase ATM (ataxia-telangiectasia, mutated), leading to phosphorylation and activation of ATM. Downstream phosphorylation and activation of TP53, together with RUNX3, activate transcription of downstream mediators as a response to injury to DNA. ${ }^{[10]}$

In our previous study with 25 patients of lung cancer (adenocarcinoma and squamous cell cancer) from Xuanwei, we found differences in 33 genes, of which LincRNA-p21 was one of them. As a result, we chose to study LincRNA-p21. In this study, we focused on three selected mediators of TP53 response: PUMA, LincRNA-p21 and PANDA. PUMA is a member of the BCL2 family and is an important mediator of apoptosis. 
Research has found that PUMA is more important than NOXA in DNA damage that induces activation of the p53-mediated mitochondrial apoptotic pathway. PUMA is located downstream of the p53 gene and has a powerful effect in promoting apoptosis and inhibition of cell growth. Low expression of PUMA exists in some tumor tissues and it is associated with the occurrence and development of tumor. Thus, increasing of PUMA can inhibit tumor growth. ${ }^{[11]}$ Long non-coding RNA p21 interacts with hnRNP-K to activate $\mathrm{p} 21$ to enforce cell cycle arrest at the G1/S phase. LincRNA-p21 is an important member of the cell cycle and its expression is directly induced by $p 53$. The result of flow cytometry and apoptosis-related enzyme activity assay confirmed LincRNA-p21 induced apoptosis in the p53 dependent pathway, and related to the formation of tumor. PANDA is a noncoding RNA that inhibits expression of apoptotic genes by sequestering NF-YA.

When DNA damage occurs, it activates expression of p53 and LincRNA-p21. In one aspect, the combination of p53 and LincRNA-p21 activates expression of PUMA. PUMA interact with members of the antiapoptotic family, and release Bax and Bak. Baxis embedded in the outer membrane of mitochondria, leading to increased permeability of the outer mitochondrial membrane. Release of cytochrome $\mathrm{C}$ apoptotic factors activates the caspase cascade, eventually leading to cell death.

This study found that expression of PANDA, LincRNA-p21 and PUMA showed no significant difference in lung tissue of male and female $(P>$ $0.05)$. Expression in squamous cell carcinoma and adenocarcinoma also showed no significant difference $(P>0.05)$, indicating that there is no gender difference or tissue specificity. Expression of LincRNA-p21 and PUMA in cancer tissues is not related but in the normal tissue it is positively correlated, perhaps due to existence of regulatory changes and disorder in cancer tissues.

In this study, we found that expression of LincRNA-p21 was higher in patients from non-Xuanwei regions with smoking index $>400$ than in patients with smoking index $<400$ and non-smokers, while in patients from Xuanwei, there was no significant difference among the three groups $(P>0.05)$. The probable reason might be that smoking is not the main reason for lung cancer in Xuanwei. The incidence of lung cancer is higher in women from Xuanwei, even though the majority of women were non-smokers. In an experiment, we exposed cells to the byproduct obtained from combustion of bituminous coal, the result of which was secretion of mediators of inflammation, damage to the cell membrane, damage to mitochondria, and mutation in nuclear DNA and mt-DNA.

Expression of LincRNA-p21 in lung cancer patients from Xuanwei was higher than non-Xuanwei patients $(P<0.05)$. We observed that alveolar epithelial cells and bronchial epithelial cells were severely damaged in patients from Xuanwei due to serious air pollution caused by burning of coal. There was also damage to DNA from pollutants released as a result of combustion of coal. Our initial research in Xuanwei found that ash and smoke of bituminous coal contained large numbers of polyaromatic hydrocarbons and nano-sized quartz particles, ${ }^{[12-14]}$ which were also found in lung tissues of lung cancer patients from Xuanwei. Polyaromatic hydrocarbons and nano-sized quartz particles can lead to damage of nuclear DNA and mitochondrial DNA. ${ }^{[15,16]}$

Expression of PUMA in lung tissues of Xuanwei patients was lower than non-Xuanwei patients $(P<0.05)$. The correlation analysis revealed that the expression of LincRNA-p21 had no correlation with expression of PUMA in normal lung tissues of Xuanwei patients, while normal lung tissues of non-Xuanwei patients were positively correlated. We considered that PUMA is a highly conserved gene in eukaryotes, possibly due to air pollution in Xuanwei causing damage to PUMA or effect the gene transcription process, but also polymorphism of LincRNA-p21 and PUMA in local population could not be ruled out, for which further research with larger sample is required.

We have successfully cultured a type of Xuanwei lung adenocarcinoma cell line (XW-05). We are now carrying out gene transfection and silencing experiments on XW-05 cells, human adenocarcinoma cells (A549), and human bronchial epithelial cells (BS2B) to observe whether XW-05 cells have specificity.

Part of this project was under the U.S. National Cancer Institute to study environmental exposure, doseeffect relationship, and epidemiology of lung cancer in Xuanwei. We are responsible for the questionnaire, collection of clinical data, specimen collection, treatment, follow-up, and prognosis. Genomic sequencing is carried out by Beijing Gene Square for National Cancer Institute. Currently, Beijing Gene Square is sequencing specimens of 421 cases, the findings of which should be reported soon.

\section{Authors' contributions}

The study's conception and design: K.Y. Yang, Z.Q. Shen

Extraction of RNA and PCR: Y.F. He, K.Y. Yang, K. Rizal Patients' enrollment and lung tissues and data's collection: K.Y. Yang, A.N. Chen, Y.C. Huang, G.Q. 
Zhao, Y.J. Lei

Paper's written: K.Y. Yang, K. Rizal

Manuscript's review and edition: K.Y. Yang, Z.Q. Shen, K. Rizal

\section{Financial support and sponsorship}

Yunnan Provincial Department of Science and Technology. Project: Use EBUS-TBNA to detect the expression of MALAT1, MYC, PVT1 genes in lymph node of NSCLC patient (No. 2016NS108); Project: Study on the mechanism of regulation of miRNAs and its target genes in Gejiu and Xuanwei NSCLC patients (No. 2014NS002).

\section{Conflicts of interest}

There are no conflicts of interest.

\section{Patient consent}

Patient consent was obtained.

\section{Ethics approval}

Ethics approval was obtained.

\section{REFERENCES}

1. Zhu C. The third cause of death review sampling survey report. Beijing: China Union Medical University Press; 2008. p. 76-84.

2. He XZ. Indoor air pollution of coal with lung cancer and genetic predisposition -- the etiology research of Xuanwei lung cancer in 22 years. J Pract Oncol 2001;16:369-70.

3. Li Y, Duan Y, Wang YM. Research progress of PUMA and its relation with lung cancer. Med Recapitulate 2010;1:1-24.

4. Cao Y, Zeng CQ. Correlation of PUMAto tumor. Basic Clin Med 2011;31:1067-9.

5. Wang KC, Yang YW, Liu B, Sanyal A, Corces-Zimmerman R, Chen
Y, Lajoie BR, Protacio A, Flynn RA, Gupta RA, Wysocka J, Lei M, Dekker J, Helms JA, Chang HY. A long noncoding RNA maintains active chromatin to coordinate homeotic gene expression. Nature 2011;472:120-4.

6. Tian L, Lucas D, Fischer SL, Lee SC, Hammond SK, Koshland CP. Particle and gas emissions from a simulated coal-burning household fire pit. Environ Sci Technol 2008;42:2503-8.

7. Tian L, Dai S, Wang J, Huang Y, Ho SC, Zhou Y, Lucas D, Koshland CP. Nanoquartz in Late Permian C1 coal and the high incidence of female lung cancer in the Pearl River Origin area: a retrospective cohort study. BMC Public Health 2008;8:398.

8. Park SY, Jeong MS, Jang SB. In vitro binding properties of tumor suppressor p53 with PUMA and NOXA. Biochem Biophys Res Commun 2012;420:350-6.

9. Happo L, Cragg MS, Phipson B, Haga JM, Jansen ES, Herold MJ, Dewson G, Michalak EM, Vandenberg CJ, Smyth GK, Strasser A, Cory S, Scott CL. Maximal killing of lymphoma cells by DNA damage-inducing therapy requires not only the p 53 targets PUMA and Noxa, but also Bim. B1ood 2010;116:5256-67.

10. Ozaki T, Nakagawara A. Role of $\mathrm{p} 53$ in cell death and human cancers. Cancers (Basel) 2011;3:994-1013.

11. Zhang KJ, Li DC, Zhu DM. PUMA expression in pancreatic cancer and its clinical significance. World Chin J Digestol 2008;16:488-92.

12. Zhao G, Huang Y, Li G, Li S, Zhou Y, Lei Y, Chen X, Yang K, Chen Y, Yang K. Subcellular distribution and genotoxicity of silica nanoparticles in human bronchial epithelial cells. Zhongguo Fei Ai Za Zhi 2013;16:117-24

13. Li G, Huang Y, Liu Y, Guo L, Zhou Y, Yang K, Chen Y, Zhao G, Lei Y. In vitro toxicity of naturally occurring silica nanoparticles in $\mathrm{C} 1$ coal in bronchial epithelial cells. Zhongguo Fei Ai Za Zhi 2012;15:561-8.

14. Yang K, Huang Y, Zhao G, Lei Y, Wang K. Expression of PAHDNA adducts in lung tissues of Xuanwei female lung cancer patients. Zhongguo Fei Ai Za Zhi 2010;13:517-21.

15. Chen XB, Huang YJ, Yang KY, Huang YC. Relationship between 8-hydroxy-2-deoxyguanosine and female lung cancer in Xuanwei region. Cancer Res Prevention Treat 2011;38:1178-80.

16. Chen AN, Yang KY, He YF, Zhao GQ, Lei YJ, Wang K. Study on mitochondrial DNA mutations and polymorphism in patients with non-small cell lung cancer in Xuanwei area. Clin Res 2014;31:205-7. 